\title{
Electrical versus optical pumping of quantum dot amplifiers
}

\author{
Berg, Tommy Winther; Bischoff, Svend; Mørk, Jesper
}

Published in:

Proceedings 27th European Conference on Optical Communication

Link to article, DOI:

10.1109/ECOC.2001.989419

Publication date:

2001

Document Version

Publisher's PDF, also known as Version of record

Link back to DTU Orbit

Citation (APA):

Berg, T. W., Bischoff, S., \& Mørk, J. (2001). Electrical versus optical pumping of quantum dot amplifiers. In Proceedings 27th European Conference on Optical Communication (Vol. 1). IEEE. https://doi.org/10.1109/ECOC.2001.989419

\section{General rights}

Copyright and moral rights for the publications made accessible in the public portal are retained by the authors and/or other copyright owners and it is a condition of accessing publications that users recognise and abide by the legal requirements associated with these rights.

- Users may download and print one copy of any publication from the public portal for the purpose of private study or research.

- You may not further distribute the material or use it for any profit-making activity or commercial gain

- You may freely distribute the URL identifying the publication in the public portal

If you believe that this document breaches copyright please contact us providing details, and we will remove access to the work immediately and investigate your claim. 


\title{
Electrical versus Optical Pumping of Quantum Dot Amplifiers
}

\author{
Tommy W. Berg, Svend Bischoff, Jesper Mørk \\ Research Center COM, DTU - Build. 345W, 2800 Lyngby, Denmark (twb@com.dtu.dk)
}

\begin{abstract}
The influence of the pumping mechanism for the dynamical properties of quantum dot amplifiers is investigated for 10,40 and $160 \mathrm{GHz}$ signals. A fast response is predicted in the case of optical pumping in the wetting layer.
\end{abstract}

\section{Introduction}

Quantum Dot (QD) amplifiers have been demonstrated to exhibit sub-picosecond gain recovery in single-pulse femtosecond pump-probe measurements $/ 1 \%$. It has later been shown that the dynamical response is limited by the carrier dynamics of the higher energy levels of the device, especially the Wetting Layer (WL) $/ 2,3 /$, and not by the recovery of the QD Ground State (GS). The maximum modulation speed of QD devices reported so far is thus on the order of $5-6 \mathrm{GHz}$ at room temperature $/ 4 /$, which is significantly slower than directly modulated bulk and Quantum Well (QW) devices. A key issue in improving the dynamical response of QD devices is to ensure faster refilling of the WL. Optical pumping directly into the WL will in this paper be shown to be an efficient method to reduce the response time of QD devices significantly compared to the case of electrical pumping. An optical holding beam has been widely used to reduce the response time of bulk and quantum well amplifiers $15 /$. Here the pump beam is used for carrier injection and not for gain clamping. The improvement of device performance using the optical pumping scheme thus opens up for the possibility of exploiting QD amplifiers for ultrafast signal processing.

\section{The Model}

The model is a generalization of the ordinary rate equation model used for bulk and QW devices. The QD's are assumed to contain two bound energy levels: a nondegenerate GS level and a doubly degenerate Excited State (ES) level (not counting spin). Escape (from ES to WL) and excitation (from GS to ES) are related to the time constants of capture (from WL to ES) and relaxation (from ES to GS) through a quasi equilibrium condition. This means that the escape and excitation times depend on the confinement energies of the GS and ES levels and the WL bandedge. Capture and relaxation processes are ascribed to phonon and carrier assisted (Auger) scattering. These processes are taken into account phenomenologically by defining WL carrier density dependent time constants. The inhomogeneous broadening of the GS and ES levels due to QD size fluctuations is taken into account by splitting the entire system of dots into a finite number of QD populations. Since the GS and ES confinement energy is different for each QD population the carrier excitation and escape times vary across the spectrum of dots. The optical field is also spectrally resolved. Our model is in principle similar to the model used by Sugawara et al. $/ 6 /$.

\section{Electrical versus optical pumping}

The QD amplifier investigated is similar to the one in ref. $/ 1 /$ and has a length of $475 \mu \mathrm{m}$. The spectrally resolved gain of the amplifier is shown in fig. 1. The solid and dashed lines correspond to the gain when pumping the amplifier electrically (EP) and optically (OP), respectively. The gain spectra are very similar in the two cases except for a slight increase around the WL edge arising from the presence of the optical pump, which is located slightly above the WL bandedge. Both the GS and ES are almost completely inverted due to the high pumping level $(7 \mathrm{~mA}$ for electrical pumping and $100 \mathrm{~mW}$ for optical pumping). The difference between electrical and optical pumping becomes visible, when a signal is injected (dotted and dashdotted lines). The ES level is almost completely depleted in case of electrical pumping, whereas the depletion of the GS and ES levels is much smaller in the case of optical pumping. The ES carriers are in both cases removed due to the rapid relaxation to the GS level. The fast capture (escape) of carriers from the WL to the ES (and vice versa) results in a modification of the entire gain spectrum. The amplification of a WDM signal in a QD amplifier would thus, like amplification in a bulk or QW amplifier, result in strong cross talk among the channels.

To illustrate the importance of the pumping scheme for the dynamical response of the amplifier we consider the injection of a short optical pulse. Fig. 2 shows the carrier dynamics for the WL, ES and GS carrier densities as function of time, when a $150 \mathrm{fs}$ pulse is amplified. The pumping conditions are identical to the case in Fig. 1.

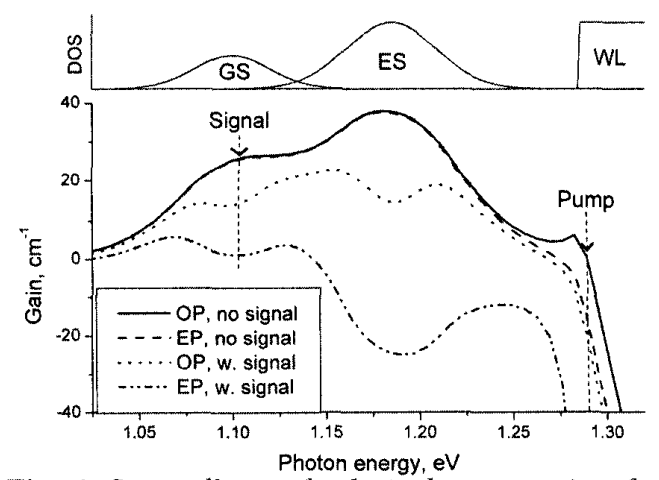

Fig. 1 Spectrally resolved single pass gain of a device under electrical (EP) and optical pumping $(O P)$, with and with out the presence of a signal at center of the GS transition. The density of states (DOS) for the system is shown at the top. 


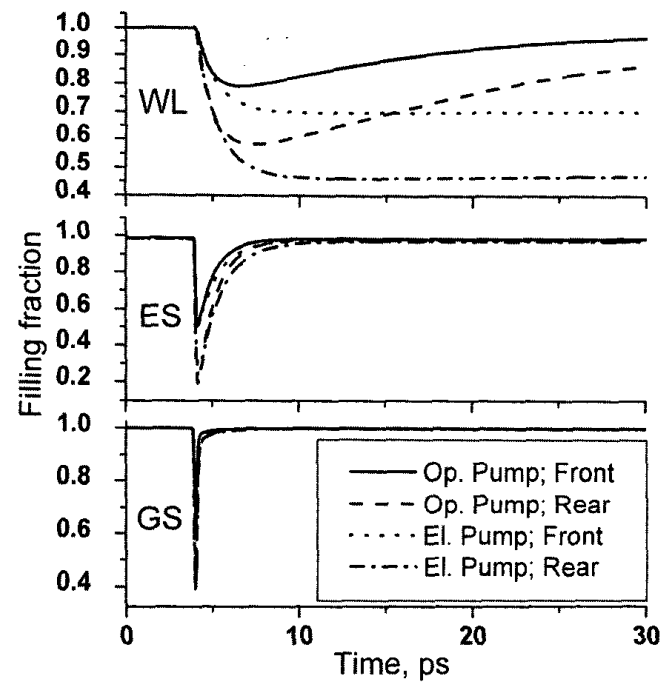

Fig. 3 Comparison of the variations of the carrier densities of the three relevant energy levels during amplification of a $150 \mathrm{fs}$ pulse when using either electrical or optical pumping. The results are shown for both the front and rear of the amplifier.

The GS population, which is initially completely inverted, basically follows the pulse envelope corresponding to a sub-picosecond relaxation time constant (ES to GS) in good agreement with experiments in $/ 1 /$ The relaxation of carriers from the ES to the GS level results in a depletion of the ES level, which is refilled within a few picoseconds by capture of carriers from the WL. The refilling of both the GS and the ES level are almost identical for electrical and optical pumping. The refilling of the WL depends strongly on the pumping scheme. For the case of electric pumping the WL recovers with a time constant given by the spontaneous recombination time of the amplifier ( $1 \mathrm{~ns})$. In contrast the recovery rate is increased many times, when the amplifier is optically pumped, due to the increased absorption of the pump beam when the WL carrier density decreases.

The optical pump power is weaker towards the end of the device compared to the input facet, since the pump is absorbed progressively during propagation. This means that the WL is refilled at a slower rate along the length of the device. The device thus becomes slower the longer it is, when pump and signal co-propagates.

The effect of the fast response time of an optically pumped QD amplifier becomes very clear, when amplifying a high frequency pulse train. Fig. 3 (A) shows the gain change of the amplifier when a $40 \mathrm{GHz}$ pulse train is injected into an electrically pumped device. The gain decreases during the first couple of pulses and then stabilizes. The steady state gain modulation is approximately $\mathrm{ldB}$. The gain variations induced by the first and last pulse of the pulse train are plotted in Fig. 3 (B) as bars. The bars can be used to evaluate the eyes in case a data signal is amplified in the QD amplifier, when the main limitation is due to patterning. The expected eye opening is given by the overlap of the gain change bars. No open eye would thus be expected for the case shown in fig. $3(\mathrm{~A})$. The calculated gain change bars at 10,40 and $160 \mathrm{GHz}$ for both electrical and optical pumping are shown in Fig. 3 (B). $\mathrm{Clear}$ and open eyes are expected for all bit rates in the case

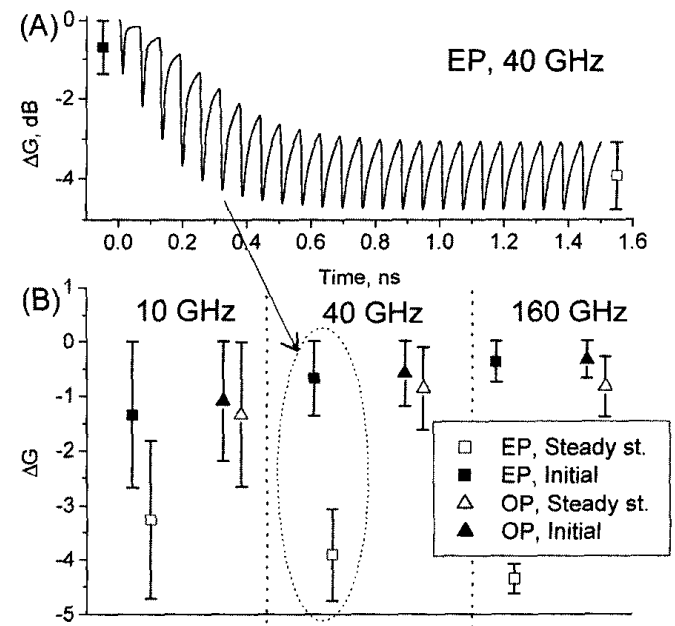

Fig. 2 (A) shows the gain change when a continuous train of short pulses with a repetition rate of 40 $\mathrm{GHz}$ is injected in the amplifier in the case of elctrical pumping. (B) compares the initial and steadystate variations of the gain over one timeslot (one pulse), which indicate the possible eye opening of the device. Results for three different bitrates for both electrical and optical pumping are given.

of optical pumping, while the electrically pumped device only is expected to result in a small open eye at $10 \mathrm{Gbit} / \mathrm{s}$. The level of gain recovery between consecutive pulses decreases as expected with increasing pulse frequency. In the example we have used parameter values corresponding to $/ 1 /$, but it is clear that for QD amplifiers to be interesting the small-signal gain should be increased significantly. It should be noted that the precise values of the gain changes depend strongly on the values of the material gain and relaxation and capture times. The point is, however, not the exact values used, but the fact that the optical pumping scheme improves the dynamical properties significantly.

\section{Conclusion}

The combination of fast relaxation and capture times and the presence of a reservoir of carriers in the WL opens up for the possibility of ultrafast gain recovery in QD devices. The strength of optical contra electrical pumping is that it reduces the bottleneck effect of a slow WL. Optical pumping thus allows significant improvement of the dynamical properties of QD devices.

\section{References}

1/ P. Borri et al., IEEE Photonics Technology Letters, Vol. 12 (2000) p. 594

12/ T. Berg et al., IEEE Photonics Technology Letters, June (2001) To be published

13/ D.G. Deppe et al., Applied Physics Letters, Vol. 77 (2000) p. 3325

14/ P. Bhattacharya et al., IEEE J. Selected Topicsin Quantum Electronics, Vol. 6 (2000) p. 426

15/ R.J. Manning et al., J. Opt. Soc. Am. B, Vol. 14 (1997), p. 3204

16/ M. Sugawara et al., Physical Review B, Vol. 61 (2000), p. 7595 